\title{
Cytologie en milieu liquide: Remettons I'église au milieu du village!
}

\author{
Prise de position en réponse à l'article de J. Obwegeser [1]
}

B. Spycher

Correspondance: Bernard Spycher, M.Sc. Cytyc Suisse S.A. Avenue du 14 Avril 7c CH-1020 Renens

E-mail:

bernard.spycher@cytyc.com Internet: www.thinprep.com
Contrairement au style de l'article précité, cette réponse présentera des preuves scientifiques concernant le frottis en couche mince (cytologie en milieu liquide) et en particulier, le ThinPrep ${ }^{\circledR}$ Pap Test. Cette réponse ne reflète pas l'opinion d'un individu ou d'un groupe. Egalement, elle a pour but de corriger certaines affirmations présentées comme des faits établis concernant Cytyc Corporation et ses filiales. Le présent document ne fera aucune allusion à des groupes, associations, individus ou à des sujets particuliers tels que TARMED.

Afin d'atteindre le premier objectif, basons nous sur les preuves cliniques relatives au ThinPrep ${ }^{\circledR}$ Pap Test. L'article susmentionné affirme que «die Evidenz betreffend höhere Sensitivität ist keinesfalls klar» et que «die einzige Evidenz, die besteht, ist, dass die Dünnschichtzytologie oder besser: 〈liquid-based cytology〉 (LBC) nicht schlechter ist als der konventionelle Abstrich, aber viel teurer und dass sehr einseitig zitiert und interpretiert wird». Il existe en fait une quantité de preuves scientifiques qui démontrent le contraire, si l'on se réfère aux études actuellement disponibles dans la littérature scientifique (peer-reviewed) relative au ThinPrep ${ }^{\circledR}$ Pap Test.

Depuis l'approbation par la FDA en mai 1996, plus de 100 études évaluant le ThinPrep ${ }^{\circledR}$ Pap Test ont été publiées dans la littérature scientifique (peer-reviewed). Les études utilisent une diversité de protocoles qui mettent en évidence un grand nombre d'avantages apportés par cette technique, y compris une augmentation du nombre de lésions dépistées, une réduction des diagnostics incertains, une amélioration de la qualité des spécimens, la possibilité d'effectuer des tests complémentaires (HPV, Chlamydia, etc.), une meilleure appréciation de la morphologie cellulaire et une amélioration du rapport coût-bénéfice.

Parmi ces études (peer-reviewed), plus de 30 ont spécifiquement comparé le ThinPrep ${ }^{\circledR}$ Pap Test au frottis conventionnel quant au dépistage des lésions du col de l'utérus et à la qualité des spécimens. Ces études comparatives représen- tent une diversité de protocoles et de cohorte de patientes. L'ensemble de toutes les populations étudiées pour tester les performances du ThinPrep $^{\circledR}$ Pap Test représente plus de 500000 patientes, et les études ont été conduites dans toutes les régions des Etats-Unis, ainsi qu'en Europe, Asie, Amérique centrale et Australie. Les résultats, remarquablement similaires, démontrent une augmentation significative de la détection de lésions cervicales au moyen du ThinPrep ${ }^{\circledR}$ Pap Test par rapport au frottis conventionnel. De plus, trois métaanalyses séparées, chacune utilisant différents critères et incluant une variété d'études, ont validé ces résultats [2-4]. En opposition à ces résultats, l'étude du Dr Obwegeser [5] n'a pas mis en évidence de supériorité du frottis en couche mince sur le frottis conventionnel. La disparité entre les résultats de l'étude Obwegeser et ceux trouvés dans le reste de la littérature médicale (30 études comparatives et 3 méta-analyses) peut éventuellement s'expliquer par la taille de la population étudiée ( $<1000$ tests ThinPrep $^{\circledR}$ ) qui n'était statistiquement pas suffisamment puissante et ni suffisante pour mettre en évidence une différence significative - qu'il s'agisse d'une augmentation ou même d'une diminution des détections. Cela peut éventuellement permettre d'expliquer le caractère de ces résultats.

Il est possible de trouver les résumés de toutes les études susmentionnées dans toutes les principales bases de données telles que Medline ou PubMed. La plupart des résumés répondent au mot-clé «ThinPrep».

Le second point de controverse dans l'article «Warum es die Dünnschichtzytologie nicht braucht!» la suggestion est faite: «Da die $\mathrm{Zu}-$ lassungsbestimmungen für Medikamente viel strenger sind als bei technischen Medizinalgeräten, dürfte sich der Einfluss der Industrie noch viel stärker auswirken.» Nous ne cautionnons pas ces déclarations. Il est important que le lecteur soit rassuré quant au fait que Cytyc n'a pas ou très peu été impliqué dans l'immense majorité des études qui ont été publiées. Seules font 
exception, par pure nécessité, les études relatives aux essais cliniques de Cytyc en vue de l'approbation par la FDA. Dans le cas d'un petit nombre d'études de santé publique, le matériel a été fourni gratuitement ou à coûts réduits, ce qui est habituel pour de telles études. Les principaux exemples d'études sponsorisées sont les études conduites par le US National Institute of Health (NIH), y compris une étude en cours au Costa Rica et la «ASCUS LSIL Triage Study (ALTS)» [6].

Finalement, l'article fait allusion à «Einfluss» exercée sur les médecins par l'industrie. Il fait également allusion à des «Gerüchte» et à de «Fehlinformationen» répandues par Cytyc Corporation. Les affirmations faites dans cet article sont injustifiées et ne tiennent pas compte de la liberté de choix et de l'indépendance des médecins. Il remet également en question la capacité de l'industrie à conduire des essais cliniques objectifs et/ou à accepter des résultats négatifs. De telles affirmations ne peuvent représenter que le seul point de vue personnel de l'auteur concernant cette question.

Les allusions aux «Gerüchte» et aux «Fehlinformationen» propagées par Cytyc dans le but de «Druck auf das BSV» constituent une autre allégation fallacieuse qui aurait pu être évitée si l'exactitude des sources avait été correctement vérifiée. Cytyc n'a fait aucune déclaration concernant un futur remboursement du frottis en couche mince par l'assurance de base. Cytyc a toutefois informé les clients qu'elle avait redéposé un dossier auprès de l'OFAS et que ce dossier devait être examinée lors de la réunion de la commission au mois de février 2004. Nous ne voyons pas en quoi cette information pourrait faire pression sur l'OFAS.

Il est regrettable que les aspects scientifiques ne soient pas seuls au centre de la controverse concernant les techniques de cytologie en milieu liquide. Mais espérons que la santé des patients, et en particulier celle des femmes, deviendra à juste titre le centre d'intérêt de toutes les décisions prises dans ce domaine.

\section{Références}

1 Obwegeser J. Warum es die Dünnschichtzytologie nicht braucht! Schweiz Ärztezeitung 2004;85(10):512-4.

2 Bernstein SJ, Sanchez-Ramos L, Ndubisi B. Liquid-based cervical cytologic smear study and conventional Papanicolaou smears: A metaanalysis of prospective studies comparing cytologic diagnosis and sample adequacy. Am J Obstet Gynecol 2001;185(2 Pt 1):308-17.

3 Abulafia O, Pezzullo JC, Sherer DM. Performance of the ThinPrep liquid-based cervical cytology in comparison with conventionally prepared Papanicolaou smears: A quantitative survey. Gynecol Oncol 2003;90(1):137-44.

4 Klinkhamer PJJM, Meerding WJ, Rosier PFWM, Hanselaar AGJM. Liquid-based cervical cytology: A review of the literature with methods of evidence-based medicine. Cancer (Cancer Cytopathol) 2003;99(5):263-71.

5 Obwegeser JH, Brack S. Does liquid-based technology really improve detection of cervical neoplasia? A prospective, randomized trial comparing the ThinPrep Pap Test with the conventional Pap test, including follow-up of HSIL cases. Acta Cytol 2001;45(5):709-14.

6 Sherman ME, Schiffman M, Cox JT for the Atypical Squamous Cells of Undetermined Significance/Low-Grade Squamous Intraepithelial Lesions Triage Study (ALTS) group. Effects of age and human papilloma viral load on colposcopy triage: data from the randomized Atypical Squamous Cells of Undetermined Significance/ Low-Grade Squamous Intraepithelial Lesion Triage Study (ALTS). J Natl Cancer Instit 2002;94(2):102-7. 